\title{
OPEN Author Correction: Fornix white matter glia damage causes hippocampal gray matter damage during age-dependent limbic decline
}

\section{Claudia Metzler-Baddeley $\mathbb{D}$, Jilu P. Mole, Rebecca Sims, Fabrizio Fasano, John Evans, Derek K. Jones, John P. Aggleton \& Roland J. Baddeley}

Correction to: Scientific Reports https://doi.org/10.1038/s41598-018-37658-5, published online 31 January 2019

This Article contains errors in Tables 2 and 3, where the uncorrected p-values are given rather than the corrected Benjamini-Hochberg adjusted p-values. As a result the Table legends,

"Summary of the effects of age on gray and white matter microstructural indices. *Controlled for intracranial volume, $* * 5 \% \mathrm{FDR}$ corrected. ISOSF $=$ isotropic signal fraction, $\mathrm{MPF}=$ macromolecular proton fraction, $\mathrm{ODI}=$ orientation dispersion index, $\mathrm{PHC}=$ parahippocampal cingulum."

should read:

"Summary of the effects of age on gray and white matter microstructural indices. *Controlled for intracranial volume, $* * 5 \%$ False Discovery Rate Benjamini-Hochberg adjusted $\mathrm{p}$-values. ISOSF $=$ isotropic signal fraction, $\mathrm{MPF}=$ macromolecular proton fraction, $\mathrm{ODI}=$ orientation dispersion index $\mathrm{PHC}=$ parahippocampal cingulum."

and

"Summary of the results of the hierarchical regression models testing for the effects of genetic and lifestyle risk variables on fornix and hippocampus mediator variables. 5\% FDR corrected p-values are highlighted in bolds."

should read:

"Summary of the results of the hierarchical regression models testing for the effects of genetic and lifestyle risk variables on fornix and hippocampus mediator variables. $\mathrm{p}_{\mathrm{BHadj}}, 5 \%$ False Discovery Rate Benjamini-Hochberg adjusted $\mathrm{p}$-values (significant $\mathrm{p}$-values are highlighted in bold). $\mathrm{BP}=$ blood pressure, $\mathrm{FH}=$ family history, ICV = intracranial volume, ISOSF $=$ isotropic signal fraction, MPF $=$ macromolecular proton fraction, WHR = waist-hip-ratio."

The correct Tables 2 and 3 appear below with their accompanying legends as Tables 1 and 2 respectively.

In addition, in Figure 3 the p-values for the following scatterplots are incorrect: Fornix ODI, LPHC R ${ }_{2}$ RPHC R , LHC R, LHC ODI and LHC $k_{f}$. As a result, the Figure legend,

"Plots the correlations and Pearson coefficients (controlled for intracranial volume) between age and white and gray matter microstructural indices. Abbr.: ICSF = intracellular signal fraction, ISOSF = isotropic signal fraction, $k_{f}=$ forward exchange rate, $\mathrm{LHC}=$ left hippocampus, $\mathrm{LPHC}=$ left parahippocampal cingulum, $\mathrm{MPF}=\mathrm{Macromolecular}$ proton fraction, $\mathrm{ODI}=$ orientation dispersion index, $\mathrm{R}=$ longitudinal relaxation rate, 


\begin{tabular}{|c|c|c|c|c|}
\hline \multicolumn{2}{|l|}{ MRI index } & \multirow{2}{*}{\begin{tabular}{l|}
$\mathbf{F}_{(2,152)}$-value $^{*}$ \\
11.9
\end{tabular}} & \multirow{2}{*}{$\begin{array}{l}\text { Benjamini-Hochberg corrected p-value** } \\
0.0002\end{array}$} & \multirow{2}{*}{$\begin{array}{l}\text { Effect size } \eta p^{2} \\
0.14\end{array}$} \\
\hline Fornix & MPF & & & \\
\hline & $k_{f}$ & 10.0 & 0.0008 & 0.12 \\
\hline & $\mathrm{R}_{1}$ & 12.4 & 0.00035 & 0.14 \\
\hline & ISOSF & 8.9 & 0.001 & 0.11 \\
\hline & ODI & 5.0 & 0.03 & 0.06 \\
\hline Left PHC & $\mathrm{R}_{2}$ & 7.5 & 0.005 & 0.09 \\
\hline Right PHC & $\mathrm{R}_{1}$ & 4.7 & 0.03 & 0.06 \\
\hline \multirow[t]{4}{*}{ Left hippocampus } & $k_{f}$ & 6.7 & 0.008 & 0.08 \\
\hline & $\mathrm{R}_{1}$ & 5.0 & 0.025 & 0.06 \\
\hline & ISOSF & 12.2 & 0.0002 & 0.14 \\
\hline & ODI & 9.8 & 0.0007 & 0.12 \\
\hline Right hippocampus & ISOSF & 7.5 & 0.004 & 0.09 \\
\hline
\end{tabular}

Table 1. Summary of the effects of age on gray and white matter microstructural indices. *Controlled for intracranial volume, **5\% False Discovery Rate Benjamini-Hochberg adjusted p-values. ISOSF = isotropic signal fraction, $\mathrm{MPF}=$ macromolecular proton fraction, $\mathrm{ODI}=$ orientation dispersion index, $\mathrm{PHC}=$ parahippocampal cingulum.

\begin{tabular}{|c|c|c|}
\hline Outcome variables & Adjusted $\mathrm{R}^{2}$ & Predictors in final regression model \\
\hline Fornix MPF & $0.24(\mathrm{p}<0.001)$ & $\begin{array}{l}\text { Age }\left(\mathrm{p}_{\text {BHadj }}<0.001\right) \\
\text { WHR }\left(\mathrm{p}_{\text {BHadj }}=0.045\right)\end{array}$ \\
\hline Fornix $\mathrm{R}_{1}$ & $0.28(\mathrm{p}<0.001)$ & $\begin{array}{l}\text { Age }\left(p_{\text {BHadj }}<0.001\right) \\
\text { ICV }\left(p_{\text {BHadj }}=0.026\right) \\
\text { Alcohol }\left(p_{\text {BHadj }}=0.03\right) \\
\text { WHR }\left(p_{\text {BHadj }}=0.04\right)\end{array}$ \\
\hline Fornix $k_{f}$ & $0.23(\mathrm{p}<0.001)$ & $\begin{array}{l}\text { Age }\left(\mathbf{p}_{\text {BHadj }}<\mathbf{0 . 0 0 1}\right) \\
\text { WHR }\left(\mathrm{p}_{\text {BHadj }}=0.07\right)\end{array}$ \\
\hline Fornix ISOSF & $0.32(\mathrm{p}<0.001)$ & $\begin{array}{l}\text { Age }\left(\mathbf{p}_{\text {BHadj }}<\mathbf{0 . 0 0 1}\right) \\
\text { ICV }\left(p_{\text {BHadj }}=0.05\right) \\
\text { Sex }\left(\mathbf{p}_{\text {BHadj }}=\mathbf{0 . 0 0 4}\right)\end{array}$ \\
\hline Right hippocampal ISOSF & $0.36(\mathrm{p}<0.001)$ & $\begin{array}{l}\text { Age }\left(\mathbf{p}_{\text {BHadj }}<\mathbf{0 . 0 0 1}\right) \\
\text { ICV }\left(p_{\text {BHadj }}=0.026\right) \\
\text { Sex }\left(p_{\text {BHadj }}=0.007\right) \\
\text { Diastolic BP }\left(p_{\text {BHadj }}=0.07\right) \\
\text { FH }\left(p_{\text {BHadj }}=0.07\right)\end{array}$ \\
\hline
\end{tabular}

Table 2. Summary of the results of the hierarchical regression models testing for the effects of genetic and lifestyle risk variables on fornix and hippocampus mediator variables. $\mathrm{p}_{\mathrm{BHad}}, 5 \%$ False Discovery Rate Benjamini-Hochberg adjusted p-values (significant p-values are highlighted in bold). BP = blood pressure, $\mathrm{FH}$ $=$ family history, $\mathrm{ICV}=$ intracranial volume, $\mathrm{ISOSF}=$ isotropic signal fraction, $\mathrm{MPF}=$ macromolecular proton fraction, WHR = waist-hip-ratio.

$\mathrm{RHC}=$ right hippocampus, $\mathrm{RPHC}=$ right parahippocampal cingulum $* * * * \mathrm{p}<0.0001, * * * \mathrm{p}<0.001, * * \mathrm{p}<0.01$, $* \mathrm{p}<0.05,5 \%$ False Discovery Rate"

should read:

"Scatterplots of the correlations and Pearson coefficients (controlled for intracranial volume) between age and white and gray matter microstructural indices. Abbr.: ICSF = intracellular signal fraction, ISOSF = isotropic signal fraction, $k_{f}=$ forward exchange rate, $\mathrm{LHC}=$ left hippocampus, $\mathrm{LPHC}=$ left parahippocampal cingulum, $\mathrm{MPF}=$ Macromolecular proton fraction, $\mathrm{ODI}=$ orientation dispersion index, $\mathrm{R}=$ longitudinal relaxation rate, $\mathrm{RHC}=$ right hippocampus, $\mathrm{RPHC}=$ right parahippocampal cingulum $* * * * \mathrm{p}<0.0001, * * * \mathrm{p}<0.001, * * \mathrm{p}<0.01$ (5\% False Discovery Rate Benjamini-Hochberg adjusted p-values).”

The correct Figure 3 and its accompanying legend appears below as Figure 1. 

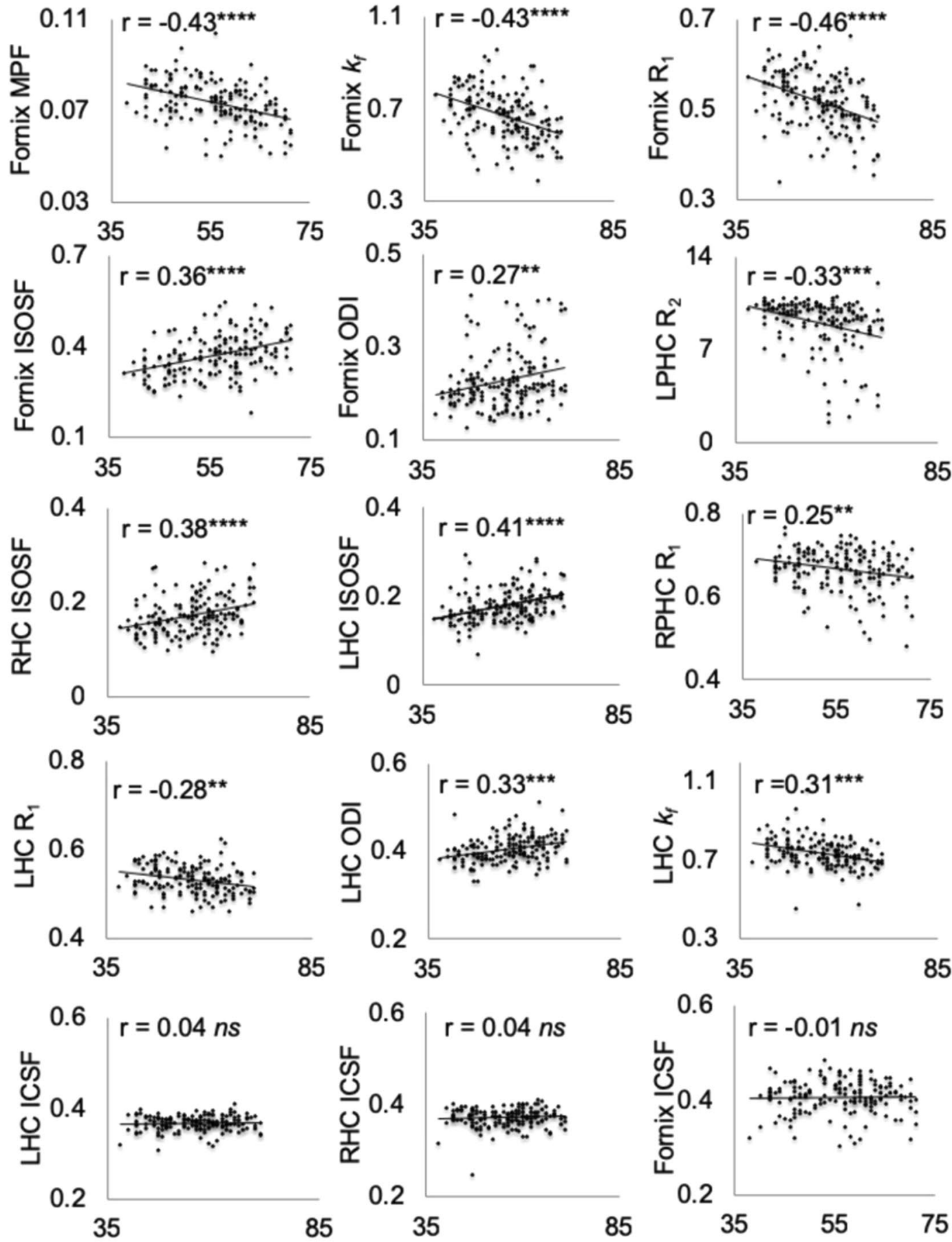

Age in years

Figure 1. Scatterplots of the correlations and Pearson coefficients (controlled for intracranial volume) between age and white and gray matter microstructural indices. Abbr.: ICSF $=$ intracellular signal fraction, ISOSF $=$ isotropic signal fraction, $k_{f}=$ forward exchange rate, $\mathrm{LHC}=$ left hippocampus, LPHC $=$ left parahippocampal cingulum, $\mathrm{MPF}=\mathrm{Macromolecular}$ proton fraction, $\mathrm{ODI}=$ orientation dispersion index, $\mathrm{R}=$ longitudinal relaxation rate, $\mathrm{RHC}=$ right hippocampus, $\mathrm{RPHC}=$ right parahippocampal cingulum $* * * * \mathrm{p}<0.0001$, ***p $<$ $0.001, * * \mathrm{p}<0.01$ (5\% False Discovery Rate Benjamini-Hochberg adjusted p-values). 
(c) (i) Open Access This article is licensed under a Creative Commons Attribution 4.0 International License, which permits use, sharing, adaptation, distribution and reproduction in any medium or format, as long as you give appropriate credit to the original author(s) and the source, provide a link to the Creative Commons license, and indicate if changes were made. The images or other third party material in this article are included in the article's Creative Commons license, unless indicated otherwise in a credit line to the material. If material is not included in the article's Creative Commons license and your intended use is not permitted by statutory regulation or exceeds the permitted use, you will need to obtain permission directly from the copyright holder. To view a copy of this license, visit http://creativecommons.org/licenses/by/4.0/.

(C) The Author(s) 2019 